\title{
Variation in age and physical status prior to total knee and hip replacement surgery: A comparison of centres in Australia and Europe.
}

\section{Authors}

Dr Ilana N Ackerman: B Physio (Hons), PhD

The University of Melbourne, Australia

Professor Paul A Dieppe: MD, FRCP

University of Oxford, United Kingdom

Associate Professor Lyn M March: MBBS, FRACP, FAFPHM

The University of Sydney, Australia

Professor Ewa M Roos: PT, PhD

University of Southern Denmark, Denmark

Dr Anna K Nilsdotter: MD, PhD

Halmstad Central Hospital, Sweden

Mr Graeme C Brown: MBBS, FRACS, FAOA

Barwon Health, Australia 
Ms Karen E Sloan: MSc

Royal Perth Hospital, Australia

Associate Professor Richard H Osborne: BSc, PhD

The University of Melbourne, Australia

\section{Address for reprint requests}

A/Prof Richard H Osborne

Centre for Rheumatic Diseases

Department of Medicine, Royal Melbourne Hospital

The University of Melbourne

Parkville Victoria 3050

AUSTRALIA

\section{Corresponding author}

A/Prof Richard Osborne

Telephone: +61 383447841

Fax: +61 393427632

Email: richardo@unimelb.edu.au

\section{Grant support}

Dr Ackerman's work was supported in part by a Henry James Williams Postgraduate Scholarship, a Melbourne Abroad Travelling Scholarship and a National Health and Medical 
Research Council of Australia Public Health Training Fellowship (\#520004). Associate Professor March’s work was supported in part by the National Health and Medical Research Council of Australia and the Lincoln Centre. Professor Roos' work was supported in part by the Swedish Research Council. Associate Professor Osborne’s work was supported in part by the Baker Trust, Buckland Foundation, Arthritis Foundation of Victoria and a National Health and Medical Research Council of Australia Career Development Award (\#400391).

Word count: 2964 


\section{Abstract}

\section{Objective}

To investigate whether variation exists in the pre-operative age, pain, stiffness and physical function of people undergoing total knee (TKR) and hip replacement (THR) at a range of centres in Australia and Europe.

\section{Methods}

Individual Western Ontario and McMaster Universities Osteoarthritis (WOMAC) Index data (scale range 0 (best) - 100 (worst)) collected within six weeks prior to primary TKR and THR were extracted from sixteen sites (total $n=2835$ ) according to specified eligibility criteria. Analysis of covariance was used to evaluate differences in pain, stiffness and physical function between centres, with adjustment for age and gender.

\section{Results}

There was marked variation in the age of people undergoing surgery across the centres (mean age 67-73 years for TKR, $\mathrm{F}_{(6,1004)}=4.21, \mathrm{p}<0.01$; mean age 63-72 years for THR, $\left.\mathrm{F}_{(14,1807)}=7.27, \mathrm{p}<0.01\right)$. Large differences in pre-operative status were observed between centres, most notably for pain (adjusted mean pain 52.5-61.1 for $\mathrm{TKR}, \mathrm{F}_{(6,1002)}=4.26$, $\mathrm{p}<0.01$; adjusted mean pain 49.2-65.7 for THR, $\left.\mathrm{F}_{(14,1802)}=8.44, \mathrm{p}<0.01\right)$ and physical function (adjusted mean function 52.7-61.4 for TKR, $\mathrm{F}_{(6,1002)}=5.27, \mathrm{p}<0.01$; adjusted mean function 53.3-71.0 for THR, $\mathrm{F}_{(14,1802)}=6.71, \mathrm{p}<0.01$ ). Large effect sizes (up to 0.98) reflect the magnitude of variation between centres and highlight the clinical relevance of these findings. 


\section{Conclusion}

The large variations in age and pre-operative status indicate substantial differences in the timing of joint replacement across the centres studied, with potential for compromised surgical outcomes due to premature or delayed surgery. Possible contributing factors include patient preferences, the absence of concrete indications for surgery and the capacity of health care systems.

Word count: 250 


\section{Introduction}

Joint replacement surgery is an effective and increasingly common intervention for end-stage knee and hip osteoarthritis. However, the specific indications for total knee (TKR) and hip replacement surgery (THR) are neither clearly-defined nor evidence-based (1) and are known to vary substantially between health professionals $(2,3)$. This lack of consensus could introduce discrepancies into the provision of joint replacement surgery both within and between countries. As early provision of surgery (for example, to younger people with less severe joint disease) or delayed surgery (undertaken in older people with more severe arthritis) can have major health consequences $(4,5)$, systematic differences between centres have important implications for clinical practice and health policy.

To date, there has been little international research comparing the pre-operative characteristics of people undergoing joint replacement; however, incidental findings from earlier studies suggest that pre-operative physical status may indeed vary between countries. People undergoing TKR or THR in Canada had higher levels of pain and poorer physical function before surgery, compared with those in the United States (6), while those undergoing TKR in the United Kingdom had poorer pre-operative physical function than their counterparts in Australia and the United States (7). As pre-operative pain and function are important predictors of outcome from joint replacement surgery (8-10), further research is essential to verify these findings and explore the extent of variation across a broad range of centres. This study investigates whether variation exists in the pre-operative age, pain, stiffness and physical function of people undergoing total knee (TKR) and hip replacement surgery (THR) at centres in Australia and Europe. 


\section{Materials and methods}

\section{Study design}

A cross-sectional analysis of available individual data extracted from research and clinical databases in Australia and Europe.

\section{Outcome measure}

The Western Ontario and McMaster Universities Osteoarthritis (WOMAC) Index is a widelyused, disease-specific measure for knee and hip osteoarthritis (11) and contains 24 items (5 pain, 2 stiffness and 17 physical function items).

\section{Available datasets}

Through consultation with international musculoskeletal researchers and clinicians it was identified that pre-operative WOMAC data for inclusion in this comparison were available from the following sites:

- Australia

- Geelong: clinical dataset

- Melbourne: research dataset

- Perth: clinical dataset

- Sydney: combined research dataset from 3 centres (containing both public and private patients)

- $\quad$ Europe

- Dundee (United Kingdom): research dataset

- EUROHIP: combined research dataset from 20 European centres 
- Lund (Sweden): research dataset

- Halmstad (Sweden): research dataset

- Hassleholm (Sweden): research dataset

Potential collaborators at each site were invited to contribute pre-operative data for this analysis and were sent a study protocol and data dictionary.

\section{Data extraction}

All of the datasets were made available in a de-identified format. Individual age, gender and WOMAC item responses were extracted from each dataset. Information on waiting time and socioeconomic status was either not routinely recorded or recorded in a way that precluded concatenation and therefore not available for this study.

\section{Eligibility criteria}

As the datasets were not collected according to a single study protocol, data from each centre were screened according to a set of eligibility criteria (specified a priori). For inclusion in the analyses, data were required to have been:

- collected from people undergoing primary unilateral TKR or THR;

- collected using the Likert version of the WOMAC Index; and

- collected within 6 weeks prior to surgery, as minimal waiting-related deterioration was anticipated to occur beyond this time point. 
Additionally, individual data were excluded if a WOMAC score could not be calculated due to an insufficient proportion of item responses (11).

After applying these eligibility criteria, it was identified that a number of centres had very small samples (combined TKR and THR samples of $n<50$ ). To minimise the likelihood of spurious results, these centres were excluded from the analyses. Details of the excluded data and reasons for exclusion are presented in the Appendix.

\section{Statistical analyses}

Analyses were undertaken using Statistical Package for the Social Sciences (SPSS) Version 14.0. WOMAC pain, stiffness and physical function subscale scores were calculated according to published scoring guidelines (11). Pain, stiffness and physical function subscale scores were transformed to a 0 (best) to 100 (worst) scale. Separate statistical analyses were undertaken for TKR and THR as the EUROHIP, Halmstad and Lund databases contained one operation type only (either TKR or THR, see Table 1). As differences in age and gender were observed between the centres (Table 2), analysis of covariance (ANCOVA) was used to evaluate whether there were differences in pain, stiffness and physical function between centres while adjusting for age and gender. Individual age and gender data were used for each ANCOVA. To explore whether there were systematic differences in the age and physical status of people undergoing surgery (for example, surgery being consistently performed on younger patients with less severe joint disease or on older patients with more severe disease), Pearson's correlation coefficient $(r)$ was used to assess the relationship between age, pain, stiffness and physical function. 
To gain further insight into the data, the magnitude of variation in pre-operative pain, stiffness and physical function across the centres was estimated using standardised effect sizes (ES). The ES statistic has been described by Cohen for use with independent samples and ESs can be categorised as small (0.20-0.49), medium (0.50-0.79) or large ( $\geq 0.8)(12)$. Data from the centre with the highest mean adjusted WOMAC score for each subscale and the centre with the lowest mean adjusted WOMAC score were used for the calculations. ESs were calculated by dividing the difference between the mean WOMAC subscale scores of the two centres by the pooled standard deviation, which was used to account for differences in sample size between centres (13). To view the observed between-centre variation in a clinical context, calculated ESs were compared with ESs for a range of commonly-used conservative osteoarthritis interventions as reported in meta-analyses and Cochrane systematic reviews. Comparisons were also made with reported ESs from studies investigating outcomes from joint replacement surgery. 


\section{Results}

\section{Demographics}

Information about the number of eligible participants, surgery dates, diagnosis and recruitment method used at each of the centres is provided in Table 1. Most participants at each centre had a diagnosis of osteoarthritis (range 91-100\%).

Age and gender data for each centre are presented in Table 2. Analysis of variance revealed marked variation in the mean age before TKR (mean age range $67-73$ years; $F_{(6,1004)}=4.21$, $\mathrm{p}<0.01$ ) and THR (mean age range 63-72 years; $\mathrm{F}_{(14,1807)}=7.27, \mathrm{p}<0.01$ ). The ratio of female to male participants also varied considerably between centres (range $42 \%-63 \%$ female for TKR and 46\%-61\% female for THR).

\section{Comparison of physical status across the centres}

Table 3 shows substantial variation in pre-operative pain and physical function across centres for people undergoing $\operatorname{TKR}\left(\mathrm{F}_{(6,1002)}=4.26, \mathrm{p}<0.01\right.$ for pain and $\mathrm{F}_{(6,1002)}=5.27, \mathrm{p}<0.01$ for physical function) and THR ( $F=8.44_{(14,1802)}, \mathrm{p}<0.01$ for pain and $\mathrm{F}_{(14,1802)}=6.71, \mathrm{p}<0.01$ for physical function). There was little variation in pre-operative stiffness across centres for people undergoing $\operatorname{TKR}\left(\mathrm{F}_{(6,1002)}=0.94, \mathrm{p}=0.46\right)$; however, greater variation in stiffness was seen among those undergoing THR $\left(\mathrm{F}_{(14,1802)}=2.81, \mathrm{p}<0.01\right)$. The extent of the observed variation in pain, stiffness and physical function is described in the following sections. As the unadjusted mean $(95 \% \mathrm{CI})$ values were comparable to the adjusted values, only the latter have been reported in Table 3. 


\section{Total knee replacement}

The mean adjusted pain scores for people undergoing TKR ranged from 52.5 to 61.1 (Table 3). People in Geelong (Australia) had, on average, the worst pre-operative pain, after adjusting for age and gender (mean adjusted physical function score 61.1). People in Perth (Australia) and Sydney (Australia) had the least pain, after adjusting for age and gender (mean adjusted physical function score 52.5 and 53.3, respectively).

There was little variation in pre-operative stiffness for people undergoing TKR, with mean adjusted scores ranging from 58.1 in Dundee (United Kingdom) to 63.3 in Geelong (Australia).

The mean adjusted physical function scores for people undergoing TKR ranged from 52.7 to 61.4. People in Geelong (Australia) and Melbourne (Australia) had the worst physical function before surgery, after adjusting for age and gender (mean adjusted physical function score 61.4 and 60.4, respectively). Those in Perth (Australia) and Dundee (United Kingdom) had the best physical function (mean adjusted physical function score 52.7 and 53.2, respectively).

\section{Total hip replacement}

The mean adjusted pain scores for people undergoing THR ranged from 49.2 to 65.7, as shown in Figure 1. People in Geelong (Australia) and Szeged (Hungary) had the worst pain prior to surgery (mean adjusted pain score 65.7 and 64.5, respectively). People in Sydney (Australia), Innsbruck (Austria) and Zurich (Switzerland) had the least pre-operative pain (mean adjusted pain score 49.2, 50.3 and 50.3, respectively). 
The mean adjusted stiffness scores for people undergoing THR ranged from 55.4 to 68.1. People in Akureyri (Iceland) and Geelong (Australia) had the worst stiffness before surgery (mean adjusted stiffness score 68.1 and 67.4, respectively), while those in Szeged (Hungary) had the least stiffness (mean adjusted stiffness score 55.4).

Figure 2 shows the mean adjusted physical function scores for people undergoing THR at each centre (range 53.3 - 71.0). People in Szeged (Hungary) and Geelong (Australia) had the worst physical function before THR (mean adjusted physical function score 71.0 and 68.5, respectively), while those in Zurich (Switzerland) and Sydney (Australia) had the best function (mean adjusted physical function score 53.3 and 54.4, respectively).

\section{Relationship between age, pain, stiffness and physical function}

Figure S1 (supplementary material) shows that for participants undergoing TKR there was no substantive relationship between age and pain $(\mathrm{r}=-0.15, \mathrm{p}<0.01)$, age and stiffness $(\mathrm{r}=-0.10$, $\mathrm{p}<0.01)$ or age and physical function $(\mathrm{r}=-0.03, \mathrm{p}=0.36)$. Similarly, for participants undergoing THR there was no relationship between age and pain $(r=0.00, p=0.89)$, age and stiffness ( $r=-$ 0.03, $\mathrm{p}=0.15$ ) or age and physical function $(\mathrm{r}=0.08, \mathrm{p}<0.01)$, as presented in Figure $\mathrm{S} 2$. These data suggest that at the centres studied, there was no systematic trend towards performing joint replacement surgery on younger patients with less severe joint disease symptoms or older patients with more severe symptoms.

\section{Estimation of the magnitude of pre-operative variation between centres}

ESs were calculated to estimate the magnitude of variation in pre-operative status across the range of centres using data from centres who had the lowest and highest WOMAC subscale 
scores according to Table 3. These analyses revealed small to medium ESs for TKR (ES for pain=0.44, ES for stiffness=0.21 and ES for physical function=0.47). For THR, medium to large ESs were found (ES for pain=0.93, ES for stiffness=0.50 and ES for physical function=0.98).

To gauge the clinical relevance of these data, the largest ESs for TKR and THR (representing the between-centre variation in physical function) were compared with published ESs for commonly-used osteoarthritis interventions. For TKR, the calculated ES for physical function of 0.47 exceeds the reported ESs for physical function following interventions such as intra-articular corticosteroid injection (ES=0.24, (14)), non-steroidal anti-inflammatory drugs (ES=0.29, (15)) and land-based exercise (ES=0.31, (16)). To interpret these data in a different clinical context, comparison with recent research (17) shows that the calculated ES of 0.47 represents approximately 30\% of the improvement in WOMAC physical function scores 6 months after TKR (ES=1.58).

For THR, the calculated ES for physical function of 0.98 exceeds the ES for physical function associated with therapies such as opioid analgesia (ES=0.31, (18)) and aquatic exercise (ES=0.76, (19)). Additionally, compared with the reported improvement in WOMAC physical function scores 6 to 12 months after THR (ES=2.40, (20); ES=2.34, (21)), the calculated ES of 0.98 represents over $40 \%$ of the reported benefit from surgery. These comparisons indicate that the magnitude of observed variation between centres can be considered substantial and clinically important. 


\section{Discussion}

This research has revealed substantial variation in the pre-operative age, pain, stiffness and physical function of people undergoing joint replacement surgery at a range of centres in Australia and Europe. The magnitude of the variability in pre-operative pain and physical function was striking (over 8 WOMAC units before TKR and over 17 WOMAC units before THR) and can be regarded as clinically meaningful, as highlighted by the calculated effect sizes. The differences in physical status were evident even after adjusting for differences in age and gender between centres. These results indicate that the timing of hip and knee replacement surgery is highly variable with respect to severity of joint disease symptoms.

The most important clinical implication is that current variations in care could lead to compromised patient outcomes for those at the extremes of the distribution. Earlier research has shown that provision of joint replacement surgery to people with greater pre-operative pain and functional impairment is associated with poorer post-operative outcomes $(8,10,22)$. Conversely, early provision of surgery to people with less severe symptoms results in smaller post-operative improvements $(10,23)$, and could represent an improper use of health resources. In almost all settings, systems are not available to determine need or urgency for joint replacement and consequently, access to surgery may be highly dependent on local customs. The absence of clear indications for joint replacement surgery is particularly relevant for countries such as the United Kingdom and Australia where general practitioners and orthopaedic surgeons act as 'gatekeepers' (1) to orthopaedic waiting lists and ultimately, to surgery.

The length of time waited for joint replacement surgery may also have contributed to the present findings, with delayed access to surgery manifesting as worse pain and poorer 
function $(10,24)$. This is highlighted by the substantial variation in pain and physical function observed between the Australian centres (Table 3). It is known that centres such as Geelong have protracted waiting times for surgery (up to 19 months for an initial appointment to see an orthopaedic surgeon and up to 27 months for surgery), whereas some of the Sydney participants were recruited from private hospitals with considerably shorter waits (in the order of days or weeks). However, waiting times are unlikely to be the only contributing factor as participants undergoing TKR in another Australian public hospital (Perth) also had considerably less pain and better physical function pre-operatively, compared with Geelong (Table 3). Although this could not be explored in the present study, the capacity of individual centres to undertake joint replacement surgery may have contributed to the observed variation, with likely differences in factors such as health care payment systems. Local rationing of surgical services due to limited resources may also impact on an individual's access to surgery so that only people with the greatest pain and functional impairment can gain access to waiting lists.

Delayed access to joint replacement surgery for particular demographic groups may lead to worse pre-operative pain and functional impairment. Disparities in access to joint replacement surgery have been identified across a range of characteristics including gender (25), age (26), racial background $(27,28)$ and socioeconomic status $(26,29,30)$, and could explain the wide variation across centres in the average age and gender ratios of people undergoing surgery in the present study. Such disparities could occur at several points in the health care process, from access to referring practitioners and orthopaedic surgeons through to entering the waiting list and finally progression along the list. Delayed access to surgery may also relate to an individual's willingness to undergo surgery, with a number of studies 
reporting differences in patient preferences for joint replacement according to gender, ethnic group and socioeconomic status (31-33).

Cultural differences in the understanding and reporting of symptoms may have also contributed to the observed variation. Although the WOMAC Index is available in a range of languages (34), it was not primarily designed for cross-cultural application and its crosscultural stability has not been reported. However, while cultural differences in the interpretation of some of the concepts (for example, pain severity) may have impacted on the findings, this is unlikely to have been a substantial contributor to the variation observed across the Australian centres.

As the international data were collected from a convenience sample of available centres rather than according to a standardised prospective protocol, it is possible that sampling variation may have contributed to differences in physical status between centres. Consecutive recruitment methods were used at each site with the expectation that the samples were broadly representative of their population base; however, it is possible that differences in recruitment rates (data not available) may have influenced this. How representative each centre is of their region or country is not known and it is possible that people with greater disease severity were recruited in some centres but not others, introducing systematic bias. Despite these limitations, this study has a number of strengths which enabled new information to be obtained. The pooling of individual data permitted WOMAC scores to be adjusted for potential confounders such as age and gender. Additionally, although a range of research and clinical datasets were used, the application of eligibility criteria allowed for some standardisation. Finally, this study extends the work of Lingard et al (7), which involved Australian participants recruited predominantly from a private hospital with short waiting 
times of less than 2 months (35); the current findings are expected to be more generalisable to people undergoing joint replacement in the Australian public health care system.

In conclusion, large between-centre variation in pre-operative age, pain, stiffness and physical function was observed in people undergoing joint replacement surgery at a range of centres in Australia and Europe. These findings have highlighted the lack of consistency between the centres studied with respect to the timing of joint replacement surgery and raise concerns regarding the quality of care and the appropriateness of services provided. Although this study did not investigate possible contributing factors, a range of patient-, medical professional- and health system-related factors have been proposed including patient preferences, the absence of concrete indications for joint replacement and the capacity of individual health care systems. Given the role of pre-operative physical status as a key predictor of joint replacement outcomes, future prospective research is warranted to uncover the origins and impact of the observed variation. 


\section{Acknowledgments}

The authors wish to thank the EUROHIP study group (see Dreinhofer et al, Annals of the Rheumatic Diseases 2006 for a full list of contributors), Beth Pollard (Scotland), the National Health and Medical Research Council (NHMRC) Arthritis COST Study Team (Australia) and the Royal Perth Hospital Joint Replacement Assessment Clinic (Australia) for contributing pre-operative data for these analyses. The authors are also grateful to Marita Cross (The University of Sydney) and Sue Williams (University of Bristol) for their assistance with data extraction.

\section{Author contributions}

Study design: Ackerman, Dieppe, March, Brown, Osborne

Acquisition of data: Ackerman, March, Roos, Nilsdotter, Brown, Sloan, Osborne Analysis and interpretation of data: Ackerman, Dieppe, March, Brown, Osborne Manuscript preparation: Ackerman, Dieppe, March, Roos, Nilsdotter, Brown, Sloan, Osborne Statistical analysis: Ackerman, Osborne 


\section{References}

1.Dieppe P, Basler HD, Chard J, Croft P, Dixon J, Hurley M, et al. Knee replacement surgery for osteoarthritis: effectiveness, practice variations, indications and possible determinants of utilization. Rheumatology 1999;38:73-83.

2.Dreinhoefer K, Dieppe P, Til S, Grober-Gratz D, Floren M, Gunther K-P, et al. Indications for total hip replacement: comparison of assessments of orthopaedic surgeons and referring physicians. Ann Rheum Dis 2006;65:1346-1350.

3.Cross WW, Saleh KJ, Wilt TJ, Kane RL. Agreement about indications for total knee arthroplasty. Clinical Orthop 2006;446:34-9.

4.Jones CA, Beaupre LA, Johnston DWC, Suarez-Almazor M. Total joint arthroplasties: Current concepts of patient outcomes after surgery. Clin Geriatr Med 2005;21:527-542.

5.Garbuz DS, Xu M, Duncan CP, Masri BA, Sobolev B. Delays worsen quality of life outcome of primary total hip arthroplasty. Clin Orthop 2006;447:79-84.

6.Fortin PR, Clarke AE, Joseph L, Liang MH, Tanzer M, Ferland D, et al. Outcomes of total hip and knee replacement. Pre-operative functional status predicts outcomes at six months after surgery. Arthritis Rheum 1999;42:1722-1728.

7.Lingard EA, Katz JN, Wright EA, Sledge CB, the Kinemax Outcomes Group. Predicting the outcome of total knee arthroplasty. J Bone Joint Surg Am 2004;86:2179-2186. 
8.Fortin PR, Penrod JR, Clarke AE, St-Pierre Y, Joseph L, Belisle P, et al. Timing of total joint replacement affects clinical outcomes among patients with osteoarthritis of the hip or knee. Arthritis Rheum 2002;46:3327-3330.

9.Nilsdotter A-K, Petersson IF, Roos EM, Lohmander LS. Predictors of patient relevant outcome after total hip replacement for osteoarthritis: a prospective study. Ann Rheum Dis 2003;62:923-930.

10.Ostendorf M, Buskens E, van Stel H, Schrijvers A, Marting L, Dhert W, et al. Waiting for total hip arthroplasty - Avoidable loss in quality time and preventable deterioration. J Arthroplasty 2004;19:302-309.

11.Bellamy N. WOMAC Osteoarthritis Index. User guide version V. Brisbane: Australia; 2002.

12.Cohen J. Statistical power analysis for the behavioral sciences. 2nd ed. New Jersey: Lawrence Erlbaum; 1988.

13.Altman DG, Machin D, Bryant TN, Gardner MJ, editors. Statistics with confidence. 2nd ed. London: BMJ Books; 2000.

14.Bellamy N, Campbell J, Robinson V, Gee T, Bourne R, Wells G. Intraarticular corticosteroid for treatment of osteoarthritis of the knee Cochrane Database of Systematic Reviews 2006, Issue 2. Art. No.: CD005328. DOI: 10.1002/14651858.CD005328.pub2 
15.Bjordal JM, Ljunggren AE, Klovning A, Slordal L. Non-steroidal anti-inflammatory drugs, including cyclo-oxygenase-2 inhibitors, in osteoarthritic knee pain: meta-analysis of randomised placebo controlled trials. BMJ 2004;329:1317: doi:10.1136/bmj.38273.626655.63.

16.Fransen M, McConnell S, Bell M. Exercise for osteoarthritis of the hip or knee. Cochrane Database of Systematic Reviews 2001, Issue 2. Art. No.: CD004376. DOI: 10.1002/14651858.CD004376

17.Escobar A, Quintana JM, Bilbao A, Arostegui I, Lafuente I, Vidaurreta I. Responsiveness and clinically important differences for the WOMAC and SF-36 after total knee replacement. Osteoarthritis Cartilage 2007;15:273-280.

18.Avouac J, Gossec L, Dougados M. Efficacy and safety of opioids for osteoarthritis: a meta-analysis of randomized controlled trials. Osteoarthritis Cartilage 2007;15:957-965.

19.Bartels EM, Lund H, Hagen KB, Dagfinrud H, Christensen R, Danneskiold-Samsøe B. Aquatic exercise for the treatment of knee and hip osteoarthritis. Cochrane Database of Systematic Reviews 2007, Issue 4. Art. No.: CD005523. DOI: 10.1002/14651858.CD005523.pub2

20.Ostendorf M, van Stel HF, Buskens E, Schrijvers AJP, Marting LN, Verbout AJ, et al. Patient-reported outcome in total hip replacement. A comparison of five instruments of health status. J Bone Joint Surg Br 2004;86:801-808. 
21.Quintana JM, Escobar A, Bilbao A, Arostegui I, Lafuente I, Vidaurreta I. Responsiveness and clinically important differences for the WOMAC and SF-36 after hip joint replacement. Osteoarthritis Cartilage 2005;13:1076-1083.

22.Nilsdotter AK, Lohmander LS. Age and waiting time as predictors of outcome after total hip replacement for osteoarthritis. Rheumatology 2002;41:1261-1267.

23.Kennedy LG, Newman J, Ackroyd CE, Dieppe PA. When should we do knee replacements? The Knee 2003;10:161-166.

24.Fielden JM, Cumming JM, Horne JG, Devane PA, Slack A, Gallagher LM. Waiting for hip arthroplasty: Economic costs and health outcomes. J Arthroplasty 2005;20:990-997.

25.Hawker GA, Wright JG, Coyte PC, Williams JI, Harvey B, Glazier R, et al. Differences between men and women in the rate of use of hip and knee arthroplasty. N Engl J Med 2000;342:1016-22.

26.Yong PFK, Milner PC, Payne JN, Lewis PA, Jennison C. Inequalities in access to knee joint replacements for people in need. Ann Rheum Dis 2004;63:1483-1489.

27.Skinner J, Weinstein JN, Sporer SM, Wennberg JE. Racial, ethnic and geographic disparities in rates of knee arthroplasty among Medicare patients. $\mathrm{N}$ Engl $\mathrm{J}$ Med 2003;349:1350-1359. 
28.Jain NB, Higgins LD, Ozumba D, Guller U, Cronin M, Pietrobon R, et al. Trends in epidemiology of knee arthroplasty in the United States, 1990-2000. Arthritis Rheum 2005;52:3928-3933.

29.Dixon T, Shaw M, Ebrahim S, Dieppe P. Trends in hip and knee joint replacement: socioeconomic inequalities and projections of need. Ann Rheum Dis 2004;63:825-830.

30.Milner PC, Payne JN, Stanfield RC, Lewis PA, Jennison C, Saul C. Inequalities in accessing hip joint replacement for people in need. European Journal of Public Health 2004;14:58-62.

31.Byrne MM, O'Malley KJ, Suarez-Almazor M. Ethnic differences in health preferences: analysis using willingness-to-pay. J Rheumatol 2004;31:1811-8.

32.Suarez-Almazor ME, Souchek J, Kelly PA, O'Malley K, Byrne M, Richardson M, et al. Ethnic variation in knee replacement. Arch Intern Med 2005;165:1117-1124.

33.Hawker GA. Who, when, and why total joint replacement surgery? The patient's perspective. Curr Opin Rheumatol 2006;18:526-530.

34.Bellamy N. WOMAC: a 20-year experiential review of a patient-centered self-reported health status questionnaire. J Rheumatol 2002;29:2473-6.

35.Lingard EA, Berven S, Katz JN. Management and care of patients undergoing total knee arthroplasty: Variations across different health care settings. Arthritis Care Res 2000;13:129136. 
Table 1 Characteristics of participating centres

\begin{tabular}{|c|c|c|c|c|c|c|c|}
\hline Region & Location (country) & Setting type & $\operatorname{TKR}(n)$ & $\operatorname{THR}(n)$ & Years of surgery & Diagnosis & $\begin{array}{l}\text { Recruitment } \\
\text { method }\end{array}$ \\
\hline \multirow[t]{4}{*}{ Australia } & Geelong & Hospital & 79 & 110 & $2002-2005$ & Mixed (97\% OA) & Consecutive \\
\hline & Melbourne & Hospital & 41 & 43 & $2002-2005$ & Mixed (90\% OA) & Consecutive \\
\hline & Perth & Hospital & 313 & 240 & $2002-2005$ & Mixed (91\% OA) & Consecutive \\
\hline & Sydney & Hospitals $^{\dagger}$ & 125 & 87 & $1994-2005$ & OA & Consecutive \\
\hline \multirow[t]{13}{*}{ Europe } & Akureyri (Iceland) & Hospital & 0 & 56 & $2004-2005$ & $\mathrm{OA}$ & Consecutive \\
\hline & Dresden (Germany) & Hospital & 0 & 114 & $2003-2004$ & OA & Consecutive \\
\hline & Dundee (UK) & Hospital & 123 & 157 & $2003-2005$ & Mixed (91\% OA) & Consecutive \\
\hline & Halmstad (Sweden) & Hospital & 0 & 87 & $1997-1998$ & $\mathrm{OA}$ & Consecutive \\
\hline & Hamburg (Germany) & Hospital & 0 & 155 & 2003 & OA & Consecutive \\
\hline & Hassleholm (Sweden) & Hospital & 252 & 390 & 2004 & OA & Consecutive \\
\hline & Innsbruck (Austria) & Hospital & 0 & 84 & $2003-2004$ & OA & Consecutive \\
\hline & Karlshamm (Sweden) & Hospital & 0 & 50 & $2003-2004$ & OA & Consecutive \\
\hline & Lund (Sweden) & Hospital & 79 & 0 & $1999-2001$ & OA & Consecutive \\
\hline & Szeged (Hungary) & Hospital & 0 & 58 & $2003-2004$ & OA & Consecutive \\
\hline & Ulm (Germany) & Hospital & 0 & 139 & $2003-2004$ & OA & Consecutive \\
\hline & Zurich (Switzerland) & Hospital & 0 & 53 & $2003-2005$ & OA & Consecutive \\
\hline & & TOTAL & 1012 & 1823 & & & \\
\hline
\end{tabular}


Table 2 Age and gender by centre

\begin{tabular}{|c|c|c|c|c|}
\hline Operation type & Centre (country) & $n$ & $\begin{array}{l}\text { Mean age in years } \\
(95 \% \mathrm{Cl})\end{array}$ & $\begin{array}{c}\text { Gender } \\
\text { (\% female) }\end{array}$ \\
\hline \multirow[t]{7}{*}{ Total knee replacement } & Dundee (UK) & 123 & 69 (67 to 70$)$ & 52 \\
\hline & Geelong (Australia) & 79 & 73 (72 to 75$)$ & 63 \\
\hline & Hassleholm (Sweden) & 252 & 71 (70 to 72$)$ & 55 \\
\hline & Lund (Sweden) & 79 & 71 (69 to 73 ) & 63 \\
\hline & Melbourne (Australia) & 41 & 67 (64 to 70$)$ & 56 \\
\hline & Perth (Australia) & 313 & 70 (69 to 71$)$ & 63 \\
\hline & Sydney (Australia) & 125 & 71 (70 to 72 ) & 42 \\
\hline \multirow[t]{15}{*}{ Total hip replacement } & Akureyri (Iceland) & 56 & 69 (67 to 72$)$ & 46 \\
\hline & Dresden (Germany) & 114 & 65 (63 to 66 ) & 56 \\
\hline & Dundee (UK) & 157 & 67 (65 to 69) & 57 \\
\hline & Geelong (Australia) & 110 & 71 (69 to 72 ) & 57 \\
\hline & Halmstad (Sweden) & 87 & 71 (69 to 73 ) & 54 \\
\hline & Hamburg (Germany) & 155 & 66 (65 to 68$)$ & 60 \\
\hline & Hassleholm (Sweden) & 390 & 70 (69 to 71$)$ & 56 \\
\hline & Innsbruck (Austria) & 84 & 63 (61 to 65$)$ & 54 \\
\hline & Karlshamm (Sweden) & 50 & 66 (63 to 69$)$ & 50 \\
\hline & Melbourne (Australia) & 43 & 67 (63 to 70$)$ & 61 \\
\hline & Perth (Australia) & 240 & 68 (66 to 69$)$ & 55 \\
\hline & Sydney (Australia) & 87 & 65 (62 to 67$)$ & 51 \\
\hline & Szeged (Hungary) & 58 & 66 (63 to 68) & 59 \\
\hline & Ulm (Germany) & 139 & 66 (64 to 68) & 53 \\
\hline & Zurich (Switzerland) & 53 & 72 (69 to 75$)$ & 55 \\
\hline
\end{tabular}




\begin{tabular}{|c|c|c|c|c|c|}
\hline \multirow[b]{2}{*}{ Operation type } & \multirow[b]{2}{*}{ Centre (country) } & \multirow[b]{2}{*}{$n^{\dagger}$} & \multirow{2}{*}{ 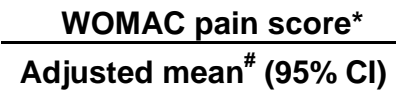 } & \multirow{2}{*}{$\begin{array}{l}\text { WOMAC stiffness score* } \\
\text { Adjusted mean }{ }^{*}(95 \% \mathrm{Cl})\end{array}$} & WOMAC physical function score* \\
\hline & & & & & Adjusted mean ${ }^{\#}(95 \% \mathrm{Cl})$ \\
\hline \multirow[t]{8}{*}{ Total knee replacement } & Dundee (UK) & 123 & 58.1 (55.1 to 61.1) & 58.1 (54.5 to 61.8$)$ & $53.2(50.2$ to 56.1$)$ \\
\hline & Geelong (Australia) & 79 & 61.1 (57.4 to 64.9$)$ & 63.3 (58.7 to 67.8$)$ & $61.4(57.6$ to 65.1$)$ \\
\hline & Hassleholm (Sweden) & 252 & 55.4 (53.3 to 57.5$)$ & $59.0(56.5$ to 61.6$)$ & 57.8 (55.8 to 59.9$)$ \\
\hline & Lund (Sweden) & 79 & 58.0 (54.2 to 61.8$)$ & 61.3 (56.8 to 65.8$)$ & 58.8 (55.1 to 62.5$)$ \\
\hline & Melbourne (Australia) & 41 & $57.4(52.1$ to 62.6$)$ & 62.2 (55.9 to 68.4$)$ & 60.4 (55.3 to 65.6$)$ \\
\hline & Perth (Australia) & 313 & 52.5 (50.6 to 54.4$)$ & 58.3 (56.1 to 60.6$)$ & 52.7 (50.8 to 54.5$)$ \\
\hline & Sydney (Australia) & 125 & 53.3 (50.3 to 56.3 ) & 59.4 (55.8 to 63.0$)$ & $55.6(52.7$ to 58.6$)$ \\
\hline & & & $F_{(6,1002)}=4.26, p<0.01^{\star *}$ & $F_{(6,1002)}=0.94, p=0.46^{\star *}$ & $\mathrm{~F}_{(6,1002)}=5.27, \mathrm{p}<0.01^{* *}$ \\
\hline \multirow[t]{16}{*}{ Total hip replacement } & Akureyri (Iceland) & 56 & 62.7 (58.1 to 67.2$)$ & 68.1 (62.9 to 73.3$)$ & 62.7 (58.3 to 67.0$)$ \\
\hline & Dresden (Germany) & 114 & 58.6 (55.5 to 61.8$)$ & 63.2 (59.6 to 66.9$)$ & 62.4 (59.3 to 65.4$)$ \\
\hline & Dundee (UK) & 157 & 60.1 (57.4 to 62.8$)$ & 58.7 (55.6 to 61.8 ) & 59.2 (56.6 to 61.8$)$ \\
\hline & Geelong (Australia) & 110 & 65.7 (62.5 to 69.0$)$ & 67.4 (63.6 to 71.1$)$ & 68.5 (65.4 to 71.6$)$ \\
\hline & Halmstad (Sweden) & 87 & 55.6 (51.8 to 59.3$)$ & 61.8 (57.5 to 66.0$)$ & 60.9 (57.4 to 64.5$)$ \\
\hline & Hamburg (Germany) & 155 & 50.9 (48.1 to 53.6$)$ & 59.1 (56.0 to 62.2 ) & 57.4 (54.8 to 60.0$)$ \\
\hline & Hassleholm (Sweden) & 390 & 59.2 (57.5 to 60.9$)$ & 60.7 (58.7 to 62.7 ) & 60.9 (59.2 to 62.5$)$ \\
\hline & Innsbruck (Austria) & 84 & 50.3 (46.6 to 54.0 ) & 58.8 (54.5 to 63.1$)$ & $55.0(51.5$ to 58.6$)$ \\
\hline & Karlshamm (Sweden) & 50 & $57.0(52.2$ to 61.8$)$ & 60.8 (55.3 to 66.3 ) & 63.7 (59.1 to 68.3 ) \\
\hline & Melbourne (Australia) & 43 & 62.4 (57.3 to 67.6$)$ & 64.2 (58.2 to 70.1$)$ & 61.8 (56.9 to 66.8$)$ \\
\hline & Perth (Australia) & 240 & 58.3 (56.1 to 60.4$)$ & 63.1 (60.6 to 65.6$)$ & 62.1 (60.0 to 64.2$)$ \\
\hline & Sydney (Australia) & 87 & 49.2 (45.5 to 52.8$)$ & 57.1 (52.9 to 61.3$)$ & 54.4 (51.0 to 57.9$)$ \\
\hline & Szeged (Hungary) & 58 & 64.5 (60.1 to 69.0$)$ & 55.4 (50.3 to 60.5$)$ & 71.0 (66.7 to 75.2$)$ \\
\hline & Ulm (Germany) & 139 & 56.7 (53.8 to 59.6) & 64.3 (61.0 to 67.6$)$ & $62.4(59.6$ to 65.1$)$ \\
\hline & Zurich (Switzerland) & 53 & 50.3 (45.7 to 55.0$)$ & $59.7(54.4$ to 65.1$)$ & 53.3 (48.9 to 57.8 ) \\
\hline & & & $F_{(14,1802)}=8.44, p<0.01^{\star \star}$ & $F_{(14,1802)}=2.81, p<0.01^{\star *}$ & $\mathrm{~F}_{(14,1802)}=6.71, \mathrm{p}<0.01^{\star *}$ \\
\hline
\end{tabular}

*Range: 0-100 for each subscale score; higher score indicates worse pain, stiffness or physical function

${ }^{\dagger}$ Missing age or gender data not able to be included in the ANCOVA analyses: $n=3$ for Halmstad (THR), $n=1$ for Hamburg (THR) and $n=1$ for Hassleholm (TKR)

\#Adjusted for age and gender

**Analysis of covariance with adjustment for age and gender 


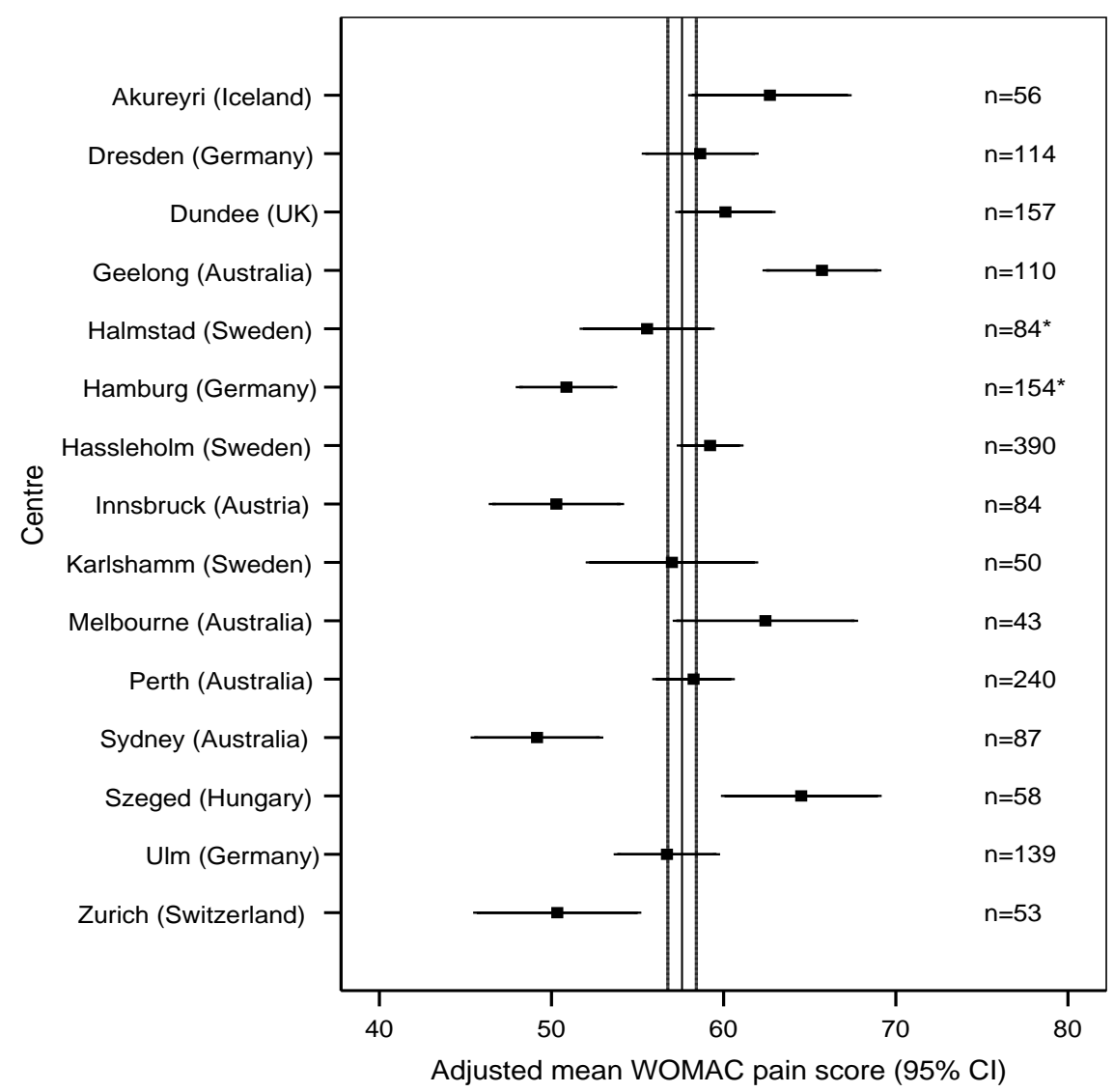

Figure 1 Pre-operative pain by centre for participants undergoing total hip replacement Scores are adjusted for age and gender; range: 0 (least pain) to 100 (worst pain)

Solid vertical line indicates pooled sample mean; dashed vertical line indicates $95 \% \mathrm{Cl}$ for pooled sample mean ${ }^{*} n$ differs to Table 1 due to missing age or gender data ( $n=3$ for Halmstad, $n=1$ for Hamburg) 


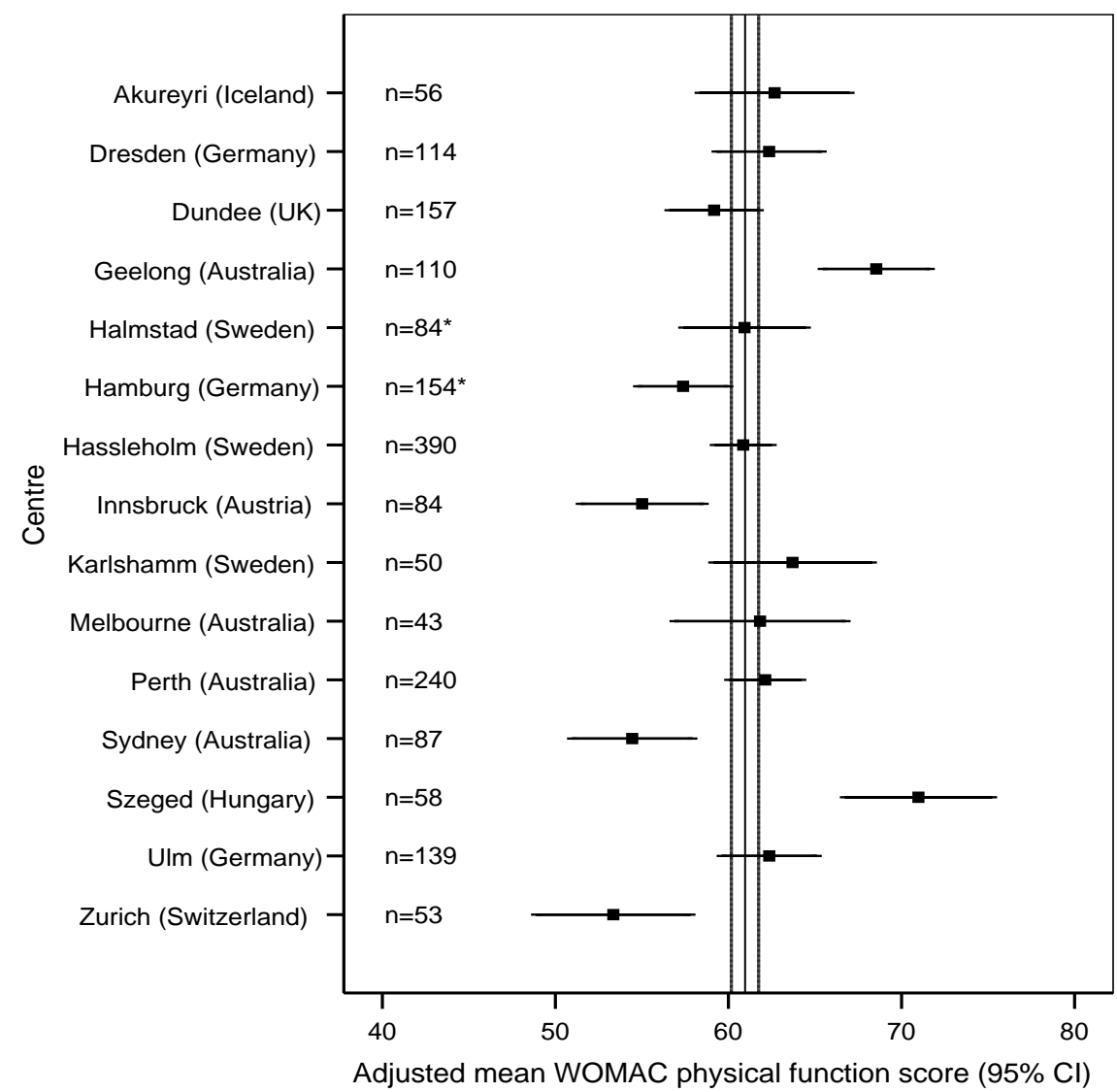

Figure 2 Pre-operative physical function by centre for participants undergoing total hip replacement

Scores are adjusted for age and gender; range: 0 (best function) to 100 (worst function)

Solid vertical line indicates pooled sample mean; dashed vertical line indicates $95 \% \mathrm{Cl}$ for pooled sample mean * $n$ differs to Table 1 due to missing age or gender data ( $n=3$ for Halmstad, $n=1$ for Hamburg) 


\section{University Library}

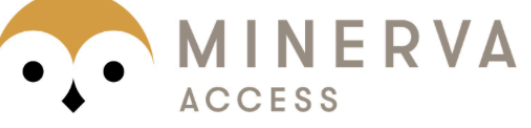

A gateway to Melbourne's research publications

Minerva Access is the Institutional Repository of The University of Melbourne

Author/s:

Ackerman, IN;Dieppe, PA;March, LM;Roos, EM;Nilsdotter, AK;Brown, GC;Sloan,

$\mathrm{KE} ;$ Osborne, $\mathrm{RH}$

Title:

Variation in Age and Physical Status Prior to Total Knee and Hip Replacement Surgery: A Comparison of Centers in Australia and Europe

Date:

2009-02-15

Citation:

Ackerman, I. N., Dieppe, P. A., March, L. M., Roos, E. M., Nilsdotter, A. K., Brown, G. C., Sloan, K. E. \& Osborne, R. H. (2009). Variation in Age and Physical Status Prior to Total Knee and Hip Replacement Surgery: A Comparison of Centers in Australia and Europe. ARTHRITIS \& RHEUMATISM-ARTHRITIS CARE \& RESEARCH, 61 (2), pp.166-173. https:// doi.org/10.1002/art.24215.

Persistent Link:

http://hdl.handle.net/11343/58919 